\title{
SISTEMA DE CLASSIFICAÇÃO EM ENFERMAGEM NANDA-NOC-NIC APLICADO AO IDOSO NO CONTEXTO DA COVID-19
}

Graziele Ribeiro Bitencourt' ORCID: 0000-0002-9130-9307

Priscilla Alfradique de Souza" ORCID: 0000-0002-4625-7552

Rosimere Ferreira Santana"' ORCID: 0000-0002-4593-3715

'Universidade Federal do Estado do Rio de Janeiro. Campus Macaé Macaé, Rio de Janeiro, Brasil.

"Universidade Federal do Estado do Rio de Janeiro. Rio de Janeiro, Rio de Janeiro, Brasil.

"'Universidade Federal Fluminense. Niterói, Rio de Janeiro, Brasil.

\section{Autor Correspondente: Graziele Ribeiro Bitencourt E-mail: grazielebitencourt@macae.ufrj.br}

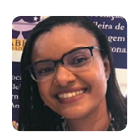

Como citar: Bitencourt GR, Souza PA, Santana RF. Sistema de Classificação em Enfermagem NANDA-NOC-NIC aplicado ao Idoso no Contexto da Covid-19. In: Santana RF (Org.). Enfermagem gerontológica no cuidado do idoso em tempos da COVID 19. Brasilia, DF: Editora ABen; 2021. 171 p. (Serie Enfermagem e Pandemias, 5). https://doi.org/10.51234/aben.21.e05.c09

\section{INTRODUÇÃO}

A COVID-19 é uma doença respiratória aguda causada pelo novo coronavírus (SARS-CoV-2), que foi declarada uma Emergência de Saúde Pública e caracterizada como uma pandemia em 11 de março de 2020(1). Nesse contexto pandêmico, o idoso apresenta maior risco de acometimento e desenvolvimento de casos graves desta doença devido às mudanças fisiológicas do processo de envelhecimento humano e detrimento do sistema imunológico, além da presença de doenças crônicas. A idade ainda é relacionada ao aumento de casos e piora do prognóstico, seja pela presença de comorbidades ou por alterações pulmonares.

Dados epidemiológicos indicam a maior ocorrência de morte por COVID-19 no idoso, sendo de 3 a $5 \%$ entre $65 \vdash 75$ anos; de 4 a $11 \%$ entre $75 \vdash 85$ anos e de 10 a $27 \%$ entre 85 anos ou mais. Além disso, há registros de $45 \%$ a mais de hospitais internações, 53\% das ocupações em Unidade de Terapia Intensiva e $80 \%$ dos óbitos ${ }^{(2)}$. Daí a necessidade de preparo no atendimento às demandas dessa clientela, tanto na prevenção quanto ao tratamento precoce.

Por outro lado, há escassez de estudos que abordem como a COVID-19 se estabelece no idoso, e sobre a efetividade das intervenções específicas do enfermeiro, a partir dos diagnósticos de enfermagem identificados. Isso aumenta a necessidade de reflexão da prática, no sentido de analisar as melhores intervenções e resultados a essa clientela, além de estimular inovações na abordagem das respostas humanas.

Nesse contexto, a enfermagem necessita estar organizada no atendimento e prestação do cuidado ao idoso no contexto da COVID-19. A Resolução COFEN n` 358 de 2009 estabelece que o processo de enfermagem é organizado nas etapas de histórico ou coleta de dados, diagnóstico, planejamento, implementação e avaliação. Trata-se de um instrumento metodológico que orienta o cuidado profissional de enfermagem e a documentação da prática profissional| ${ }^{(3)}$. Esta documentação pode ter seu registro 
padronizado por meio de sistema de classificações como a Nanda Internacional (Nanda-I) para diagnósticos de enfermagem, Classificação de Resultados de enfermagem (NOC) para resultados e a Classificação de intervenções de enfermagem (NIC) para intervenções.

Entretanto, particularidades são apresentadas nessas classificações, tanto nos contextos quanto nos diferentes ciclos vitais que carecem do olhar do enfermeiro para implementação do cuidado de qualidade e com a maior brevidade possível. É preciso expertise das áreas, como a enfermagem gerontológica, para dar atenção às especificidades no idoso no levantamento das repostas humanas apresentadas na COVID-19. Sendo assim, quais os diagnósticos, resultados e intervenções de enfermagem identificáveis no idoso no contexto da COVID-19, com base nas classificações NANDA-I, NOC e NIC?

\section{OBJETIVO}

Descrever o processo de enfermagem ao idoso no contexto da COVID-19 mediante uso dos sistemas de classificação NANDA-NOC-NIC

\section{MÉTODO}

Trata-se de um estudo descritivo, tipo análise reflexiva da literatura à luz da Teoria os Padrões Funcionais de saúde, conforme Figura 1.

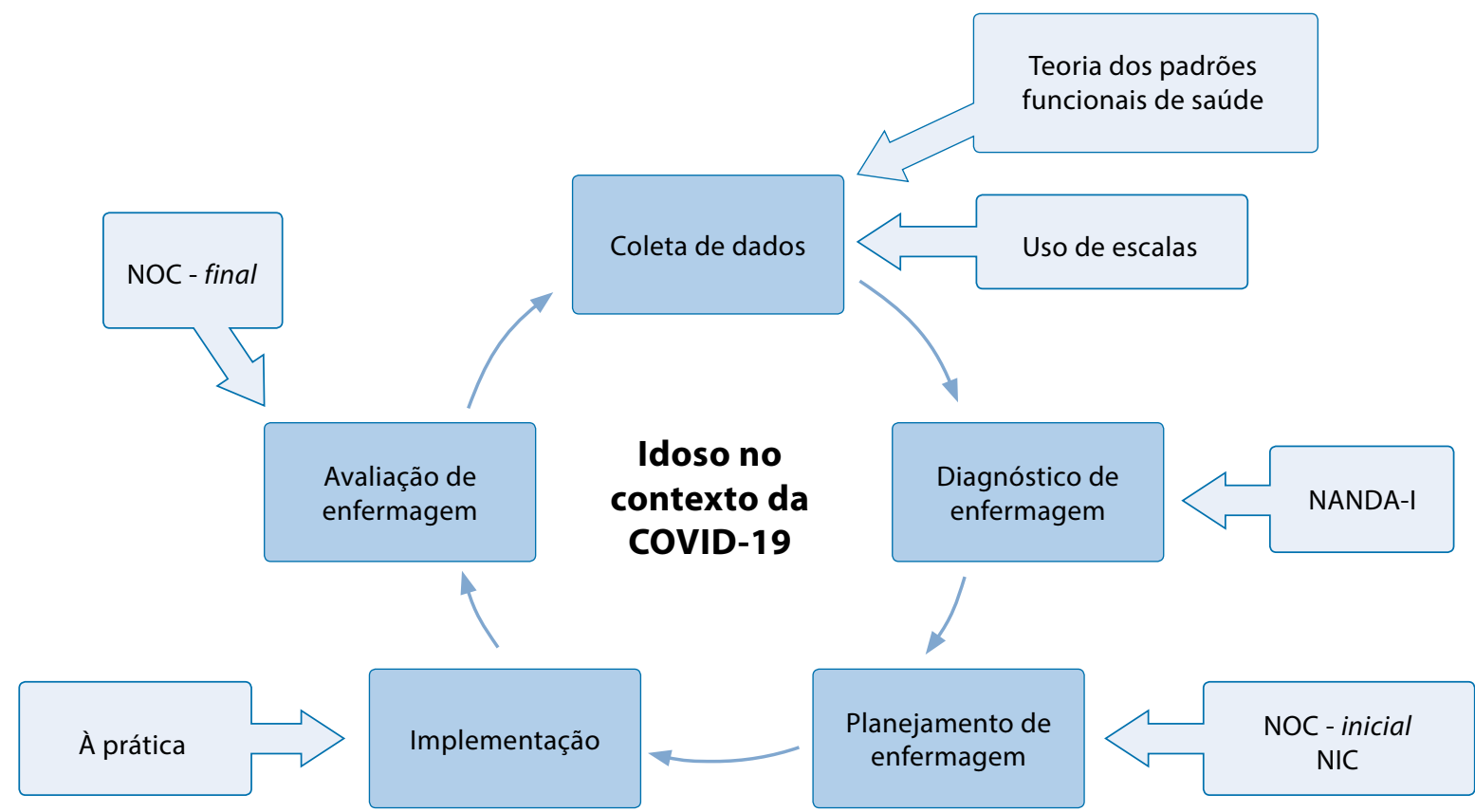

Figura 1 - Processo de enfermagem do idoso à luz da Teoria dos padrões Funcionais de Saúde no contexto da pandemia COVID-19.

O idoso com COVID-19 requer considerações de aspectos gerontológicos característicos em um continuum de avaliação. Nesse sentido, há necessidade de avaliação das especificidades do processo de envelhecimento para o reconhecimento das respostas humanas iniciais da COVID-19, as quais precisam ser consideradas em todas as fases do processo de enfermagem. 


\section{Teoria de Padrões Funcionais de Saúde}

O uso de teorias sustenta a aplicação do processo de enfermagem. Nesse contexto, a Teoria dos Padrões Funcionais de Saúde foi desenvolvida pela Marjory Gordon em 1982 e consiste em onze categorias nominais, representadas em áreas básicas de saúde que auxiliam na coleta dos dados para a elaboração de diagnósticos de enfermagem. Essas áreas fundamentam a prática de enfermagem e podem ser descritas a partir de dados clínicos obtidos pela história e exame físico realizado pelo enfermeiro, além de exames laboratoriais ${ }^{(4)}$.

Tem-se, portanto, essa proposta de abordagem de coleta e reflexão de informações no idoso com COVID-19 a partir dos padrões de: percepção e controle de saúde, nutricional-metabólico, eliminações, cognitivo-perceptivo, autopercepção e autoconceito, desempenho de papel e relacionamento, sexual-reprodutivo, resposta e tolerância ao estresse, crença e valor, atividade e exercício e sono e repouso ${ }^{(5)}$.

\section{Histórico de enfermagem}

O histórico de enfermagem remete a coleta de dados como um processo deliberado, sistemático e contínuo, realizado com o auxílio de métodos e técnicas variadas com a finalidade de obtenção de informações sobre a pessoa, família ou coletividade humana e suas respostas em um dado momento do processo saúde e doença ${ }^{(3)}$.

No contexto da COVID-19, três (3) dimensões precisam ser consideradas na avaliação de enfermagem ao idoso. A primeira é a clínica, que abrange o histórico de saúde-doença na avaliação ampliada e centrada e o exame físico, buscando identificar a presença de agravos, doenças crônicas e agudas, hábitos, antecedentes familiares, quantidade e tipos de medicamentos utilizados. A segunda é a psicossocial, que considera os aspectos relacionados à cognição, memória, humor, comportamentos e saúde mental, atentando para situações de sofrimento psíquico e transtornos mentais estabelecidos, suporte familiar e social, de questões econômicas, culturais, ambientais, étnico-raciais, de gênero. A terceira é a dimensão funcional, que avalia de forma objetiva a capacidade ou não de realizar atividades da vida diária, utilizando diferentes habilidades ${ }^{(6)}$.

A coleta de dados iniciais pode ser subjetiva, através de informações coletadas na entrevista a partir do relato do idoso ou familiar; e objetiva, obtidos por meio do exame físico, imagem ou laboratoriais. Um relato também pode indicar necessidade de avaliação mais objetiva: um idoso, por exemplo, com queixa de dispneia precisa de uma avaliação física respiratória aprofundada para análise geral do problema. Para tanto, um exame físico minucioso pode ser necessário como parâmetro inicial para estabelecimento do status de melhora ou piora na avaliação, última etapa do processo de enfermagem ${ }^{(7)}$.

Instrumentos padronizados ou esquemas de avaliação formal completos também podem nortear a avaliação dos dados objetivos e subjetivos. O uso de escalas padronizadas pode incentivar práticas consistentes, ajudar a garantir a segurança e permitir a detecção de alterações. Podem, em geral, auxiliar na avaliação da capacidade funcional, ou seja, na investigação sobre a independência e/ou autonomia na realização de atividades necessárias ou desejáveis na vida diária.(7).

Dessa forma, pode-se avaliar a capacidade funcional e obter informações para o planejamento de cuidados a longo prazo. Para tanto, é necessária a investigação inicial das atividades da vida diária (AVDs), em três níveis de complexidade.

1. A primeira delas é a atividade básica da vida diária (ABVDs) que compreende as habilidades necessárias para gerenciar as necessidades físicas básicas de uma pessoa, incluindo higiene ou cuidados pessoais, vestir-se, ir ao banheiro, transferir ou ambular e comer.

2. A segunda abrange as atividades instrumentais ou intermediárias da vida diária (AIVDs) que compreendem a capacidade de pensamento mais complexas, incluindo habilidades organizacionais, como 
transporte, compras; gerenciamento e finanças; refeições; limpeza doméstica e manutenção em casa; comunicação e gerenciamento de medicamentos.

3. E a terceira é a atividade avançada da vida diária (AAVDs) que compreende atividades sociais, produtivas, físicas e de lazer. Alguns instrumentos foram desenvolvidos e validados para a língua portuguesa a fim

\section{Básica}

- Índex de Katz

- Índice de Barthel

- Medida de Independência Funcional (MIF)

\section{Instrumental}

- Escala de Lawton e Brody

- Questionário de Atividades Instrumentais de Pfeffer

\section{Avançada}

- Mini exame do estado mental

- Teste de fluência verbal

- Teste de desenho do relógio

- Escala de depressão geriátrica

Figura 2 - Principais instrumentos de avaliação das atividades de vida diária no idoso.

No processo avaliativo, o ideal é a aplicação desses instrumentos, entrevista e avaliação objetiva na consulta de enfermagem inicial. Recomenda-se no mínimo, um instrumento de cada nível de atividade de vida diária aplicado em conjunto com as demais técnicas. Entretanto, é necessário o envolvimento do idoso e da família no processo para se estabelecer um parâmetro inicial e a base para os diagnósticos e intervenções nas próximas etapas do processo de enfermagem.

\section{Diagnósticos de enfermagem no idoso na COVID-19}

Os diagnósticos de enfermagem são definidos como um processo de interpretação e agrupamento dos dados coletados na histórico, que culminam com a tomada de decisão sobre os conceitos que representam, as respostas da pessoa, família ou coletividade humana em um dado momento do processo saúde e doença. Constituem a base para a seleção das ações ou intervenções com as quais se objetiva alcançar os resultados esperados ${ }^{(3)}$.

Quadro 1 - Diagnósticos de Enfermagem possíveis em idosos no contexto da COVID 19

\begin{tabular}{|l|l|}
\hline Domínios (NANDA-) & Diagnósticos de enfermagem \\
\hline \multirow{2}{*}{ Promoção da saúde } & Risco de síndrome do idoso frágil (00231) \\
\cline { 2 - 2 } & Proteção ineficaz (00043) \\
\hline \multirow{2}{*}{ Nutrição } & Nutrição desequilibrada: menor do que as necessidades corporais(00002) \\
\cline { 2 - 3 } & Risco de volume de líquido desequilibrado(00025) \\
\hline
\end{tabular}


Continuação do Quadro 1

\begin{tabular}{|l|l|}
\hline Domínios (NANDA-) & Diagnósticos de enfermagem \\
\hline \multirow{4}{*}{ Eliminação e troca } & Diarreia(00013) \\
\hline \multirow{4}{*}{ Percepção/cognição } & Insônia(00095) \\
\cline { 2 - 2 } & Padrão respiratório ineficaz (00032) \\
\cline { 2 - 3 } Papeis e relacionamentos & Intolerância à atividade (00092) \\
\hline \multirow{5}{*}{ Segurança/proteção } & Risco de confusão aguda (00173) \\
\hline \multirow{5}{*}{ Conforto } & Risco de infecção(00004) \\
\cline { 2 - 3 } & Risco de tromboembolismo venoso(00268) \\
\cline { 2 - 2 } & Desobstrução ineficaz de vias aéreas (00031) \\
\cline { 2 - 3 } & Risco de contaminação (00180) \\
\cline { 2 - 3 } & Hipertermia (00007) \\
\hline & Dor aguda (00132) \\
\cline { 2 - 3 } & Náusea (00134) \\
\cline { 2 - 3 } & Isolamento social (00053) \\
\hline
\end{tabular}

Os diagnósticos de enfermagem do idoso no contexto da COVID-19 organizados a partir dos domínios da NANDA-(1), estão representados no Quadro 1.

Devido aos estudos da COVID-19 abordarem os sinais e sintomas iniciais e complicações da doença, as respostas humanas que evidenciam os diagnósticos de enfermagem aparecem, principalmente, nos aspectos clínicos. Entretanto, os diagnósticos de Proteção Ineficaz, Interação social prejudicada e Isolamento Social, podem ser identificados e são referentes as respostas comportamentais, inerentes a situação especifica do lidar com o contexto pandêmico.

\section{Intervenções e resultados de enfermagem}

A etapa de planejamento no processo de enfermagem é definida como determinação dos resultados que se espera alcançar e das ações ou intervenções de enfermagem que serão realizadas face às respostas da pessoa, família ou coletividade humana em um dado momento do processo saúde e doença, identificadas na etapa de diagnóstico de enfermagem (Resolução COFEN 358 de 2009). Trata-se, portanto, da etapa inicial de utilização das classificações $\mathrm{NOC}^{(9)}$ e $\mathrm{NIC}^{(10)}$ para resultados e intervenções de enfermagem, respectivamente.

Quadro 2 - Resultados e intervenções de Enfermagem prioritárias em idosos no contexto da COVID 19

\begin{tabular}{|l|l|l|}
\hline Diagnósticos (Nanda-I) & Resultados (NOC) & Intervenções (NIC) \\
\hline $\begin{array}{l}\text { Risco de síndrome do idoso frágil } \\
(00231)\end{array}$ & $\begin{array}{l}\text { Autocontrole de doença crônica } \\
\text { Controle de Riscos: Processo Infeccioso }\end{array}$ & $\begin{array}{l}\text { Consulta por Telefone } \\
\text { Ensino: Processo da Doença }\end{array}$ \\
\hline Proteção ineficaz (00043) & Conhecimento: promoção da saúde & $\begin{array}{l}\text { Ensino: indivíduo } \\
\text { Controle de infecção }\end{array}$ \\
\hline $\begin{array}{l}\text { Nutrição desequilibrada: menor } \\
\text { do que as necessidades corporais } \\
(00002)\end{array}$ & Estado Nutricional & $\begin{array}{l}\text { Aconselhamento Nutricional } \\
\text { Controle da Nutrição }\end{array}$ \\
\hline
\end{tabular}


Continuação do Quadro 2

\begin{tabular}{|c|c|c|}
\hline Diagnósticos (Nanda-I) & Resultados (NOC) & Intervenções (NIC) \\
\hline $\begin{array}{l}\text { Risco de volume de líquido } \\
\text { desequilibrado (00025) }\end{array}$ & Equilíbrio Hídrico & Monitoração Hídrica \\
\hline Diarreia (00013) & Eliminação intestinal & Controle da Diarreia \\
\hline Insônia (00095) & $\begin{array}{l}\text { Sono } \\
\text { Nível de fadiga }\end{array}$ & $\begin{array}{l}\text { Controle do Ambiente: Conforto } \\
\text { Melhora do sono }\end{array}$ \\
\hline Padrão respiratório ineficaz (00032) & Estado Respiratório: Ventilação & $\begin{array}{l}\text { Controle de vias aéreas } \\
\text { Monitorização respiratória } \\
\text { Oxigenoterapia }\end{array}$ \\
\hline Intolerância à atividade (00092) & Assistência no Autocuidado & $\begin{array}{l}\text { Autocuidado: Atividades da Vida } \\
\text { Diária (AVD) }\end{array}$ \\
\hline Risco de confusão aguda (00173) & Cognição & Proteção contra Infecção \\
\hline Interação social prejudicada (00052) & $\begin{array}{l}\text { Envolvimento social } \\
\text { Comunicação }\end{array}$ & Apoio da família durante tratamento \\
\hline Risco de infecção (00004) & Gravidade da Infecção & $\begin{array}{l}\text { Controle de Imunização/Vacinação } \\
\text { Controle de Infecção }\end{array}$ \\
\hline $\begin{array}{l}\text { Risco de tromboembolismo venoso } \\
(00268)\end{array}$ & $\begin{array}{l}\text { Conhecimento: prevenção de trombos } \\
\text { Controle de Riscos: Trombo }\end{array}$ & $\begin{array}{l}\text { Controle da Terapia Trombolítica } \\
\text { Precauções contra Embolia }\end{array}$ \\
\hline $\begin{array}{l}\text { Desobstrução ineficaz de vias aéreas } \\
(00031)\end{array}$ & $\begin{array}{l}\text { Estado Respiratório: Permeabilidade das Vias Aéreas } \\
\text { Controle de Riscos: Processo Infeccioso }\end{array}$ & $\begin{array}{l}\text { Controle de Vias Aéreas } \\
\text { Estimulação da Tosse } \\
\text { Monitorização Respiratória }\end{array}$ \\
\hline Risco de contaminação (00180) & $\begin{array}{l}\text { Controle de Risco Comunitário: Doenças } \\
\text { Contagiosas }\end{array}$ & $\begin{array}{l}\text { Controle do Ambiente: Comunidade } \\
\text { Proteção Contra Infecção }\end{array}$ \\
\hline Hipertermia (00007) & Termorregulação & Regulação da Temperatura \\
\hline Dor aguda (00132) & $\begin{array}{l}\text { Controle da Dor } \\
\text { Nível de Dor }\end{array}$ & Controle da Dor \\
\hline Náusea (00134) & Controle de Náusea e Vômitos & Controle da náusea \\
\hline Isolamento social (00053) & $\begin{array}{l}\text { Envolvimento social } \\
\text { Apoio Social }\end{array}$ & $\begin{array}{l}\text { Melhora da Socialização } \\
\text { Melhora do sistema de apoio }\end{array}$ \\
\hline
\end{tabular}

Com base nesse levantamento, podem ser traçados possíveis resultados esperados e intervenções com base na NOC e NIC, respectivamente, conforme Quadro 2.

Após a elaboração dos resultados e intervenções de enfermagem, faz-se necessária a etapa de implementação, para a execução de ações e a finalização com o monitoramento da efetividade das ações através da avaliação. No contexto do idoso, as especificidades do processo de envelhecimento precisam ser consideradas para o estabelecimento das metas e prazos cabíveis a clientela, bem como intervenções específicas, ou seja, não adaptadas do adulto.

\section{CONSIDERAÇÕES FINAIS}

A implantação do processo de enfermagem do idoso requer o estabelecimento de diagnósticos condizentes com a clientela, seguidos de metas e intervenções específicas. Neste estudo foi possível refletir sobre esses diagnósticos, resultados e intervenções relacionados ao cuidado ao idoso no contexto da COVID-19 através do uso das classificações NANDA-I, NOC e NIC. Entretanto, pontua-se a necessidade de estudos que apontem a efetividade de cada intervenção, embasada no diagnóstico de enfermagem para assim contribuir 
com a qualificação da assistência e o avanço da prática baseada em evidência.

\section{AGRADECIMENTO}

\section{Ao Departamento Científico de Enfermagem Gerontológica da ABEn Nacional.}

\section{REFERÊNCIAS}

1. Centers for Disease Control and Prevention. Severe outcomes among patients with Coronavirus Disease 2019 (COVID-19) - United States, February 12 - March 16, 2020. Morbidity and Mortality Weekly Report (MMWR)[Internet]. 2020 [cited 2021 Jan 09];69(12):343-6. Available from: https://www.cdc.gov/mmwr/volumes/69/wr/mm6912e2.htm

2. Strabelli TMV, Uip DE. COVID-19 and the Heart. Arq Bras Cardiol. 2020;114(4):598-600. https://doi.org/10.36660/ abc.20200209

3. Conselho Federal de Enfermagem (COFEN). Resolução no. 358/ 2009. Dispõe sobre a Sistematização da Assistência de Enfermagem e a implementação do Processo de Enfermagem em ambientes públicos ou privados, em que ocorre o cuidado profissional de Enfermagem, e dá outras providências. Diário Oficial da União 23 out 2009.

4. Herdman TH, Kamitsuru S. Diagnósticos de enfermagem da NANDA Internacional: definições e classificação 2018-2020. 11 ed. Porto Alegre: Artmed; 2018.

5. Gordon M. Nursing diagnosis: process and application. St. Louis (US). McGraw-Hill; 1982.

6. Ministério da Saúde (BR). Secretaria de Atenção à Saúde. Orientações técnicas para a implementação de linha de cuidado para atenção integral à saúde da pessoa idosa no sistema único de saúde - SUS [Internet]. Brasília: Ministério da Saúde; 2018[cited 2021 Jan 09]. Available from: https://bvsms.saude.gov.br/bvs/publicacoes/linha_cuidado_atencao_pessoa_ idosa.pdf. Acesso em 09 de janeiro de 2020.

7. Spirgiene L, Brent L. Comprehensive geriatric assessment from a nursing perspective. In: Hertz K, Santy-Tomlinson J, (Eds). Fragility fracture nursing: perspectives in nursing management and care for older adults. Springer: Cham, 2018.

8. Dias EG. Atividades avançadas de vida diária e incidência de declínio cognitivo em idosos: Estudo SABE. Cad Saúde Pública. 2015;31(8). https://doi.org/10.1590/0102-311X00125014

9. Johnson M, Moorhead S, Maas ML, Swanson E. Classificação dos Resultados de Enfermagem - NOC. 5. ed. São Paulo: Elsevier; 2016.

10. Bulechek GM, Butcher HK, Dochterman J, Wagner CM. Classificação das Intervenções de Enfermagem - NIC. 6. ed. São Paulo: Elsevier, 2016. 